\title{
MODELLING REFRIGERATING COMPARTMENT OF FROST-FREE DOMESTIC REFRIGERATORS
}

\author{
J.E. JARAMILLO ${ }^{1,2, \text { * }}$ \\ J. RIGOLA ${ }^{1}$ \\ I. RODRIGUEZ ${ }^{1}$ \\ C. OLIET ${ }^{1}$
}

\author{
${ }^{1}$ Centre Tecnològic de Transferència de Calor (CTTC) \\ Universitat Politècnica de Catalunya (UPC) \\ ETSEIAT, C. Colom 11, 08222 Terrassa (Barcelona), Spain \\ ${ }^{2}$ Escuela de Ingeniería Mecánica \\ Universidad Industrial de Santander (UIS) \\ Cra 27 calle 9, Bucaramanga, Colombia
}

Received: 03/12/11

Accepted: 09/03/12 *to whom all correspondence should be addressed: e-mail: jejarami@uis.edu.co

\begin{abstract}
This paper is focussed on the study of the air movement and temperature distribution inside refrigerating compartment of household frost-free refrigerators. It is well known that the correct air circulation and temperature distribution inside the refrigerated chamber are two of the most important factors that affect refrigerator efficiency. In the frost-free refrigerators the cooled air is supplied directly inside the fresh food and vegetable cabinets. Therefore, studies intended to establish the actual air flow and temperature distributions inside these cabinets are relevant in order to improve temperature homogeneity and to reduce energy consumption. The methodology applied in this work is based on the numerical simulation of the cabinets by means of computational fluid dynamics. Turbulence is solved by means of a Large Eddy Simulation model. Unsteady threedimensional numerical studies are carried out, simulating the cooling process starting from a uniform warm temperature inside the refrigerator. Furthermore, the influence of the location of inlet and outlet ports is also investigated.
\end{abstract}

KEYWORDS: turbulence modelling, LES, CFD, symmetry-preserving discretization, air movement, refrigerator.

\section{INTRODUCTION}

Nowadays, final users are not only concerned about the refrigerator capability to preserve perishable food products fresh, which is its basic function, but also about the refrigerator performance referring to energy saving (Fukuyo et al., 2003). It is well known that the correct air circulation and temperature distribution inside the refrigerated chamber are the most important factors that affect refrigerator efficiency (Meng and $\mathrm{Yu}, 2009$ ). Furthermore, a rapid cooling is also desirable to avoid a premature deterioration of the food products (Gupta et al., 2007). In the frost-free refrigerators the cooled air is supplied directly into the fresh food and vegetable cabinets. Therefore, studies intended to establish the actual air flow and temperature distributions inside these cabinets are relevant in order to improve temperature homogeneity and reduce energy consumption. Hence, this paper is focussed on the study of temperature distribution and air movement inside the household frost-free refrigerators.

This type of refrigerators has been working for the last two decades. However, only a few studies dealing with the thermo-fluid dynamics behaviour inside this kind of refrigerators can be found in the technical literature. An example of the experimental work carried out to study the performance of the supply-air openings is that recently presented by Meng and Yu (Meng and Yu, 2009). Numerical studies to model refrigerator behaviour assuming steady state and laminar regime have been done by Gupta et al. (Gupta et al., 2007). They also conducted experiments to validating their numerical results. They found a qualitative agreement between the numerical predictions and the experimental results. Moreover, Fukuyo et al. (Fukuyo et al., 2003) and Ding et al. (Ding et al., 2004) studied the 
flow in turbulent regime by means of RANS $k-\varepsilon$ models. Unlike, these papers, in this work the turbulence is solved by means of advanced LES models. Furthermore, unsteady three-dimensional numerical studies, simulating the cooling process starting from a uniform warm temperature inside the refrigerator are carried out. Moreover, the influence of the location of inlet and outlet ports is considered.

The numerical methodology applied in the present paper is based on the finite volume technique. The governing partial differential equations are converted into algebraic ones using unstructured meshes. Second-order schemes are used for transient and spatial discretization. Local grid refinement is applied (Lehmkuhl et al., 2007). Furthermore, the mathematical formulation used in this work is based on symmetry-preserving discretization of the governing equations, which assures that some important properties of the Navier-Stokes equations are retained in the discretization process (Verstappen and Veldman, 2003).

\section{MATHEMATICAL FORMULATION}

The volume-filtered Navier-Stokes equations of the fluid flow (continuity, momentum and energy), used in LES simulations, can be written as follows:

$$
\begin{aligned}
& \frac{\partial \overline{\mathrm{u}}_{i}}{\partial x_{i}}=u \\
& \frac{\partial \overline{\mathrm{u}}_{i}}{\partial t}+\overline{\mathrm{u}}_{j} \frac{\partial \overline{\mathrm{u}}_{i}}{\partial x_{j}}=-\frac{1}{\rho} \frac{\partial \bar{p}}{\partial x_{i}}+\frac{\partial}{\partial x_{j}}\left(2 v \bar{S}_{i j}-\tau_{i j}\right)-\rho g_{i} \beta\left(\bar{T}-T_{r e f}\right) \\
& \frac{\partial \bar{T}}{\partial t}+\overline{\mathrm{u}}_{i} \frac{\partial \bar{T}}{\partial x_{i}}=\frac{\partial}{\partial x_{i}}\left(\frac{\lambda}{\rho c_{p}} \frac{\partial \bar{T}}{\partial x_{i}}-h_{i}\right)
\end{aligned}
$$

where $h_{i}$ is the subgrid-scale (sgs) heat flux; and $\tau_{i j}$ is the sgs stress tensor. These new unknowns appear in the filtering process as a result of the non-linear character of the convective term. They must be somehow determined in order to close the system of equations. $\tau_{i j}$ is responsible for the energy transfer between the filtered and resolved eddies. Furthermore, $h_{i}$ represents the turbulent transport of the temperature due to the unresolved (subgrid) velocity field.

The sgs stress tensor is generally modelled using the strain rate tensor of the resolved field $\bar{S}_{i j}=\frac{1}{2}\left(\frac{\partial a_{i}}{\partial x_{i}}+\frac{\partial a_{i}}{\partial x}\right)$ and the subgrid eddy viscosity, $v_{g g s}$, as follows:

$\tau_{i j}=\overline{u_{i} u_{j}}-\overline{\mathrm{u}}_{i} \overline{\mathrm{u}}_{j}=-2 v_{\mathrm{ggs}} \bar{S}_{i j}+\frac{1}{3} \tau_{k \mathrm{k}} \delta_{i j}$

Different models, which are explained below, can be used to model the subgrid eddy viscosity. Furthermore, for $h_{i}$ the selected models use the simple eddy diffusivity approach that can be written as:

$h_{i}=-\frac{v_{\text {ggs }}}{\operatorname{Pr}_{\text {sgs }}} \frac{\partial \bar{T}}{\partial x_{i}}$

where $F r_{g g z}$ is the subgrid Prandtl number.

\section{Subgrid-scale model}

A suitable approach for the subgrid eddy viscosity is needed to model turbulent scales that cannot be resolved. The simplest model is the Smagorinsky model (Smagorinsky, 1963). In the Smagorinsky's model, the subgrid viscosity is proportional to the subgrid characteristic length scale $\Delta$ and to a characteristic turbulent velocity.

$\left.v_{a g s}=\left(C_{s} \Delta\right)^{2} \sqrt{2 \bar{S}_{i j} \bar{S}_{i j}} \equiv\left(C_{s} \Delta\right)^{2} \mid \bar{S}\right], \quad \Delta=\mathrm{V}^{1 / 3}$

One shortcoming of this model is that the Smagorinsky coefficient $C_{s}$ is a constant that must be tuned. Furthermore, this model needs a damping function to correct the near-wall behaviour of the subgrid viscosity. Therefore, in order to overcome these deficiencies Nicoud and Ducros (Nicoud and Ducros, 1999) proposed a model known as wall adapting local eddy viscosity (WALE) model. This model accounts for the effects of both the strain and the rotation rate of the smallest resolved turbulent fluctuations. Moreover, it reproduces correctly the subgrid eddy viscosity behaviour near 
solid walls, and it does not require a second filtering operation to evaluate Smagorinsky coefficient, thus it saves computing time. The WALE model, which is selected in this work to approximate subgrid eddy viscosity, reads:

$$
\begin{aligned}
& v_{s g s}-\left(C_{w} \Delta\right)^{2} \frac{\left(\bar{S}_{i j}^{d} \bar{S}_{i j}^{d}\right)^{\frac{3}{2}}}{\left(\bar{S}_{i j} \bar{S}_{i j}\right)^{\frac{5}{2}}+\left(\bar{S}_{i j}^{d} \bar{S}_{i j}^{d}\right)^{\frac{5}{4}}} \\
& \bar{S}_{i j}^{d}=\frac{1}{2}\left(g_{i j}^{2}+g_{j i}^{2}\right)-\frac{1}{3} \delta_{i j} g_{k j}^{2} \\
& g_{i j}=\frac{\partial \bar{u}_{i}}{\partial x_{j}}, \quad g_{i j}^{2}=g_{i k} g_{k j} \\
& C_{w}=0.33, \quad p_{r_{s g}}=0.4
\end{aligned}
$$

\section{NUMERICAL METHODOLOGY}

The proposed methodology is based on the numerical simulation of the cabinet by means of computational fluid dynamics and heat transfer (CFD\&HT). A new general purpose CFD\&HT code, called TermoFluids (Lehmkuhl et al., 2007), is used in this work. The code uses efficient algorithms, which work adequately on slow networks of personal computers clusters. At is was commented, turbulence is solved by means of the WALE LES model (Sagaut, 2001). Governing partial differential equations are converted into algebraic ones using unstructured collocated meshes, symmetrypreserving discretization (Verstappen and Veldman, 2003), and considering Boussinesq assumption. Pressure-velocity coupling is solved by means of fully explicit fractional step algorithm. A third-order gear like scheme is used for time integration (Fishpool and Leschziner, 2009). Conservative schemes, which preserve kinetic energy, are applied for the spatial discretization. Local refinement of the grid, where large thermal and velocity gradients exist, is applied. Partitioning of the computational domain is carried out by means of MeTIS software. For the solution of the pressure (Poisson) equation, an iterative conjugate-gradient (CG) method with a diagonal scaling preconditioner is used (Borrell et al., 2007).

\section{ILLUSTRATIVE NUMERICAL RESULTS \\ 4.1 Configuration analysed}

In Figure 1 a schematic representation of the refrigerating compartment studied is presented. As it was already explained, the first $t=15 \mathrm{~s}$ of the cooling process starting from a uniform warm temperature inside the refrigerator is numerically studied. At the beginning of the simulation a constant temperature $\left(T_{0}\right)$ of $283 \mathrm{~K}$ is assumed in the whole space for the cases analysed. Uniform velocity and temperature $\left(T_{\text {in }}\right)$ profiles of cooled air are given at the inlet openings, which are located in the rear wall. An inlet mass flow rate $\left(\dot{m}_{\text {in }}\right)$ of $2.0 \times 10-3 \mathrm{~kg} / \mathrm{s}$ at $\mathrm{T}_{\text {in }}=263 \mathrm{~K}$ is imposed. Non-slip condition is used at walls and shelves. Furthermore, they are considered adiabatic. In the air return grill a pressure outflow boundary condition is assumed. Moreover, two inlet ports distributions are studied while inlet mass flow rate is kept constant. A summary of the numerical and physical parameters taken into account in this work is presented in Table 1.

Firstly, inlet openings plotted with solid line are used (referred as case 1). Then, two inlet openings are added, which are depicted with dashed line in Figure 1 (referred as case 2). Return grill, which is placed behind vegetable compartment, and the rest of the geometry is similar for the two cases. A fully three dimensional unstructured grid with approximately 4100000 control volumes (cv) is used. The mesh is refined near the air inlet, the outlet and the solid walls, taking care of capturing steep gradients near these places.

Table 1. Physical and numerical simulation parameters

\begin{tabular}{ccccccc}
\hline$m_{\text {in }}\left[\mathrm{kg} \mathrm{s}^{-1}\right]$ & $\mathrm{T}_{\mathrm{o}}[\mathrm{K}]$ & $\mathrm{T}_{\text {in }}[\mathrm{K}]$ & $\mathrm{t}[\mathrm{s}]$ & Grid $[\mathrm{cv}]$ & Case 1 & Case 2 \\
\hline $2.0 \times 10-3$ & 283 & 263 & 15 & 4100000 & 6 inlets & 8 inlets \\
\hline
\end{tabular}




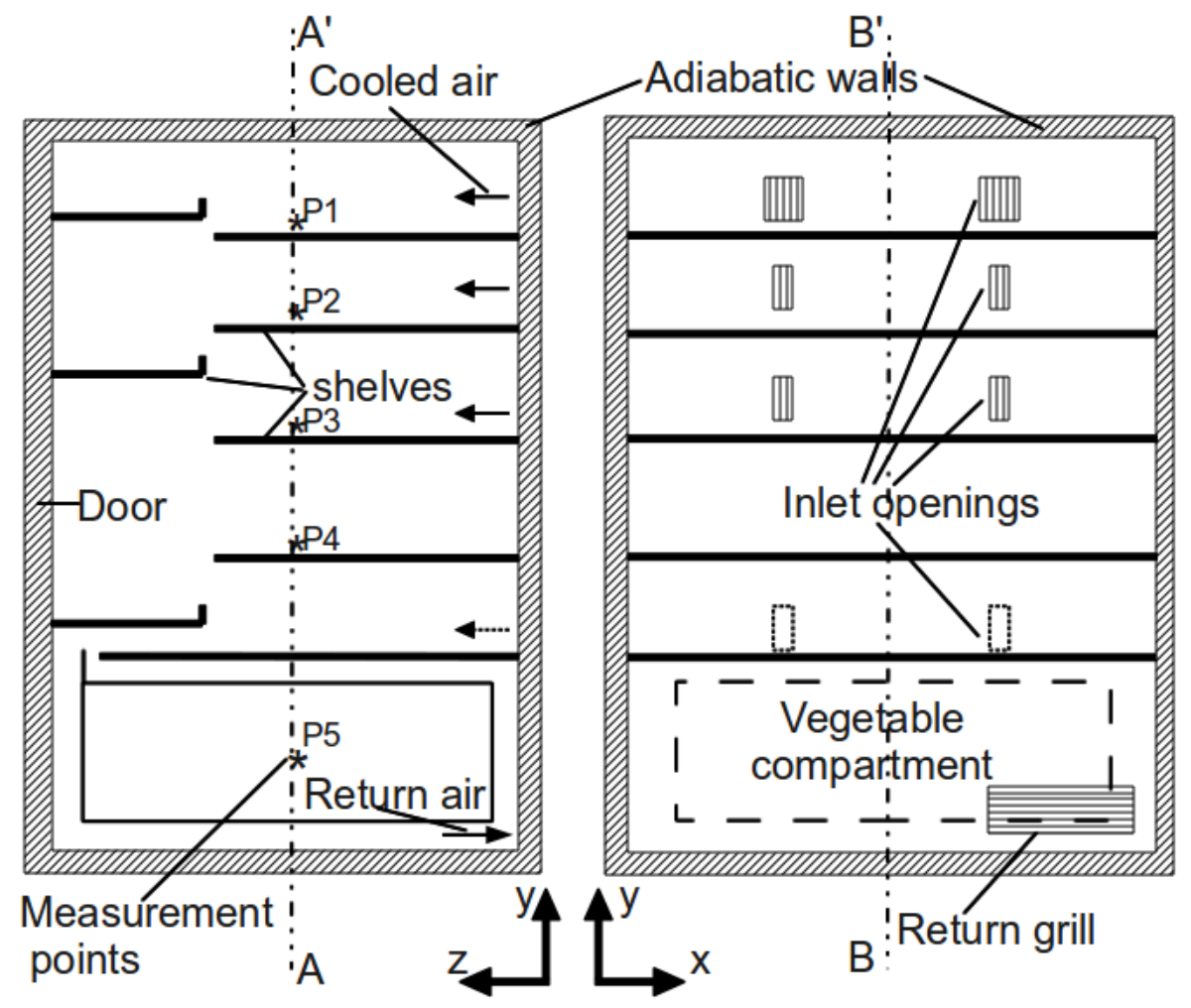

Figure 1. Refrigerator layout

\subsection{Results and discussion}

This section is dedicated to the presentation of numerical results obtained by the authors in order to characterise fluid-dynamic and thermal fields of the refrigerating compartment of a household frostfree refrigerator. Even though the grid used in this simulation can be considered adequate, it is still advisable to carry out a more detailed verification process to determine the grid independence of the numerical results. However, given the complexity of the studied configuration a compromise between computational time and desired accuracy should be achieved. Therefore, results hereafter presented should be taken as a good qualitative approximation (see also (Jaramillo et al., 2010)).

Firstly, the geometry with six inlet openings, case 1, is analysed. Numerical results for this configuration are presented in Figure 2. In this Figure, temperature distribution after 15s is shown. Red colour represents profiles where temperature has decreased one degree, i.e. air is at $282 \mathrm{~K}$. As it can be seen, in some regions temperature has not diminished even by $1 \mathrm{~K}$ after $15 \mathrm{~s}$.

Air flow in this refrigerating compartment is given by mixed convection. At the beginning, the air flows into the space impinging the door shelves. Then, part of it gains heat from the surroundings, becoming lighter and flowing up due to buoyancy effects. Straight after, recirculating it mixes with the cold air stream emanating from the inlets and flows down, entering the second shelve. At this point, part of the air settles down in the upper part of this space. Whereas, the other part is recirculated when it mixes with the inlet stream of this shelf. Finally, the combined air flows down through the gap between the door and the shelf. The process is repeated for the third shelf. However, below the fourth shelf there are not inlet openings and most of the air is heated by the surroundings here and it creates a recirculation. Only a small part of the cold air flows down towards the lid of vegetable compartment.

In an effort to improve the air temperature distribution homogeneity in the vertical direction, two new inlet openings are included below the fourth shelf from the top. Their location is depicted with dashed line in Figure 1 (case 2). The mass flow is kept constant and similar to the one used in the case 1 configuration. The most remarkable difference can be observed near the bottom part of the cabinet in Figure 3. As a consequence of the new inlet ports, cooled air now produces a decrease of the temperature near the vegetable compartment and the space below the fourth shelf, where the new inlets are located. Furthermore, in this Figure is easy to see that the temperature has reduced below, in the front of, and around the vegetable compartment. 


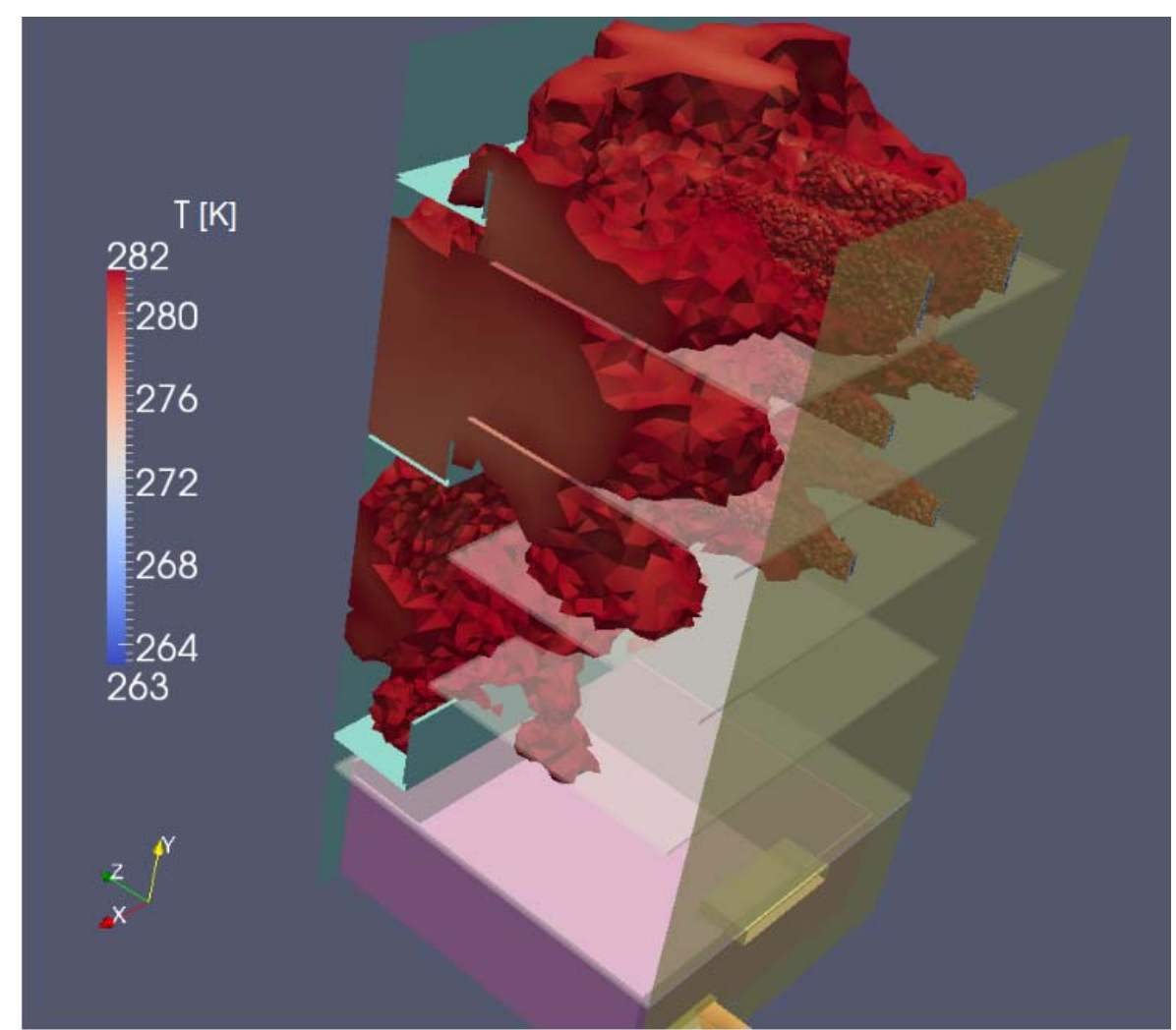

Figure 2. Case 1. Temperature distribution after $15 \mathrm{~s}$ of having started the cooling process

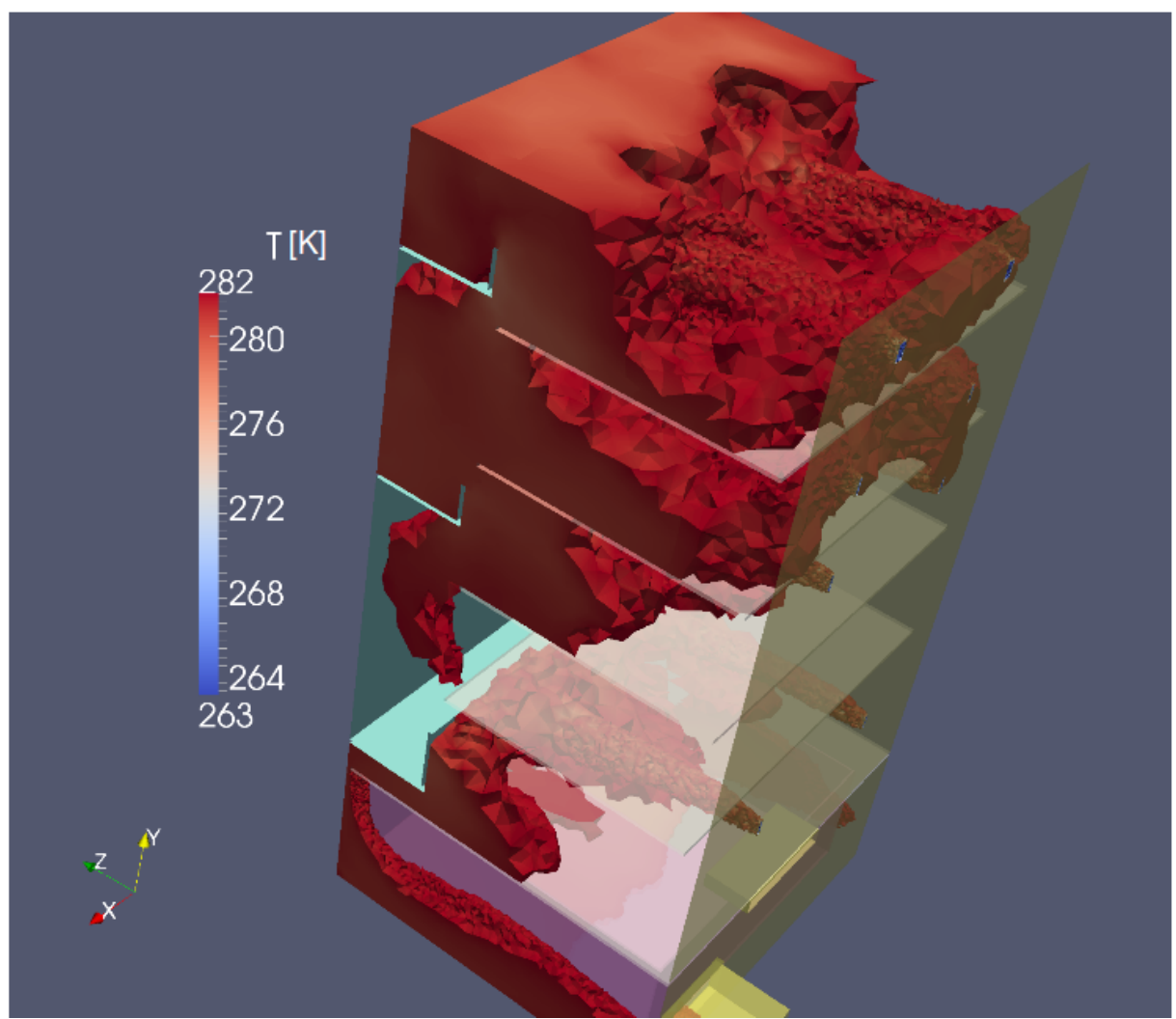

Figure 3. Case 2: Temperature distribution after 15s of having started the cooling process

However, the cooled air has not been introduced within vegetable box. Moreover, it seems that due to the new inlet velocity, the air temperature in the top part of the refrigerating compartment has decreased in case 2 a bit more than in case 1. 
The temperature distribution for case 1 seen from the back part (section AA') of the refrigerator, after the same 15s of simulation, is presented in Figure 4(a). As it can be seen, the temperature is not uniform in the horizontal direction, especially in the three upper shelves, where the inlet ports are located. Apart from the inlet jets, the coolest regions are observed near the walls, between second and third shelves. Furthermore, the temperature in the upper part is the lowest, and it increases towards the bottom part of the refrigerating compartment. At this time, the entire vegetable compartment remains approximately at the initial temperature for case 1 configuration. Moreover, the cold air begins to flow down towards the vegetable compartment by the central part of the cabinet in horizontal direction.

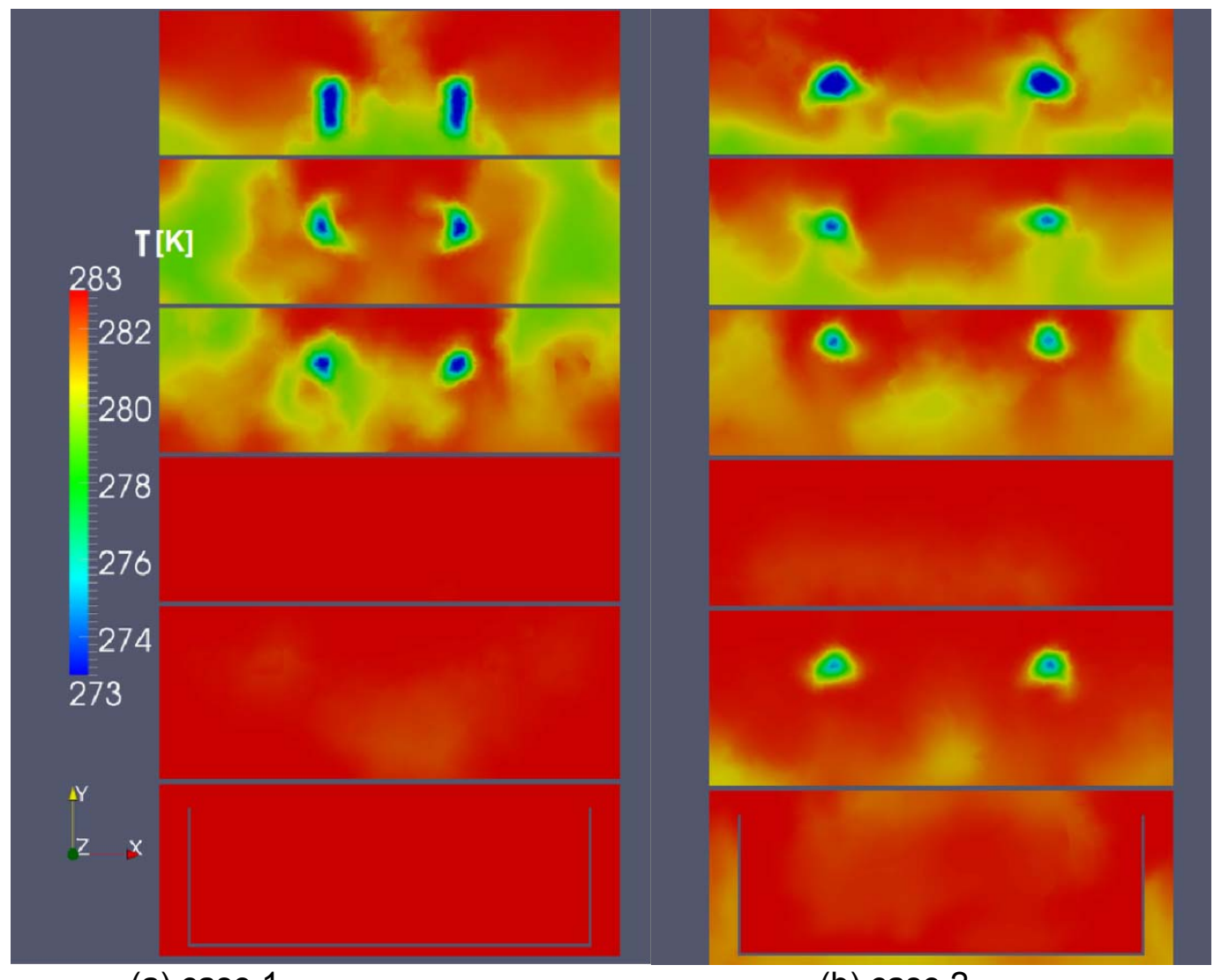

(a) case 1

(b) case 2

Figure 4. Temperature distribution in the central cross section $\mathrm{AA}^{\prime}$

In order to compare the differences between the inlets configurations studied, a rear view of the temperature distribution for case 2 (section $A A^{\prime}$ ) is shown in Figure 4(b). As it can be seen, in the upper part of the refrigerating cabinet, the temperature distribution is more regular in the horizontal direction. A temperature reduction can be observed over the first and the second shelves. For the case 2 new temperature profiles are also observable below fourth shelf. Furthermore, now the air flows down more uniformly through the gap between door and cabinet shelves. In this Figure is also possible to observe as the cold air flows around the vegetable box towards the outlet grid reducing in this way the temperature in this region, whereas, the air within vegetable box remains still almost unchanged.

Figure 5 shows the temperature contours within the refrigerating compartment at the symmetry plane BB' for both case 1 (a) and case 2 (b). It can be seen from this Figure that using case 1 the lowest temperature is obtained near the door between the first and the second shelves from the top. Hence, a reduction of approximately $7 \mathrm{~K}$ is observed in this zone. Furthermore, the temperature decreases faster in the portion adjacent to the door. On the other hand, using case 2 the temperature reduction is less than using case1, especially in the region near the door. However, a lower temperature is observed in a larger zone within the refrigerating compartment. Furthermore, the temperature in the region over the trays has reduced more using case 2, even on the lid vegetable compartment, and around it. In spite of the temperature reduction is less using case 2 than using case 1, a more homogeneous distribution, which covers a larger zone, is obtained. 
Nevertheless, the temperature in the space between the third and the fourth shelves remains almost unaffected in both case 1 and case 2. The addition of the two inlet ports in case 2 located in the space above the lid of vegetable compartment, as depicted in Figure 1, shows that: i) air on the fifth shelf becomes colder faster than in case 1, due to the inlets position; ii) the air in the bottom part of the cabinet is colder in case 2 , and it exits colder than in case 1.

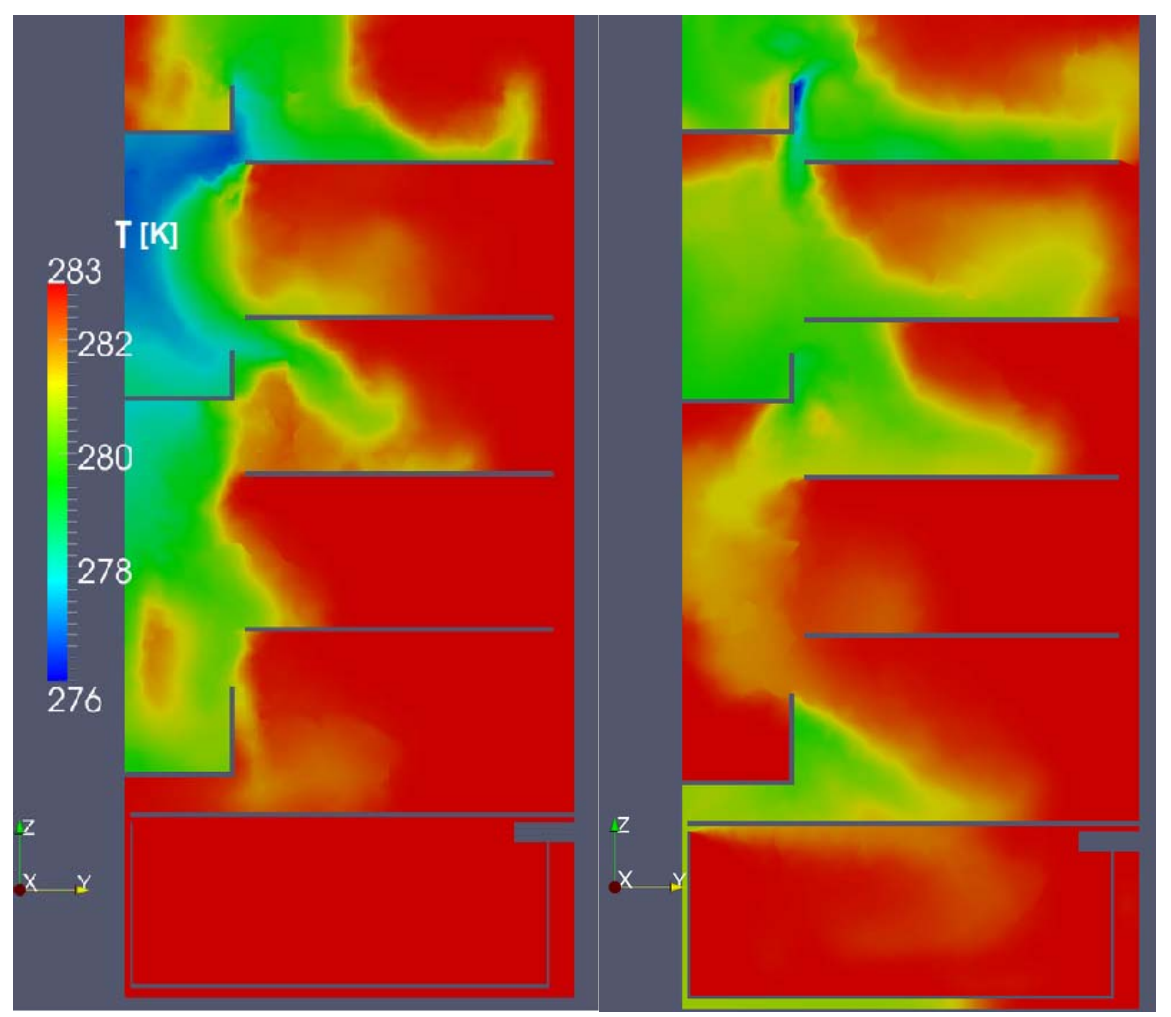

(a) case 1

(b) case 2

Figure 5. Temperature distribution on the symmetry central plane BB'

Temperature variations along the central vertical axis are shown in Figure 6. The lowest temperature is observed at P1 location and it increases as one move downward along this axis. For all points studied the temperature is lower using case 2. Moreover, the temperature at points P4 and P5 using case 1 has not changed yet. After the first $5 s$ of simulation only the temperature at P1 of case 2 has decreased considerably. Because of the first cold air impingement against the top door drawer, a recirculation of cold air is created between the two upper inlet jets. Then the air gains heat from surrounding, it becomes warmer and it fills the upper space. In this way a lower temperature at the top shelf is maintained.
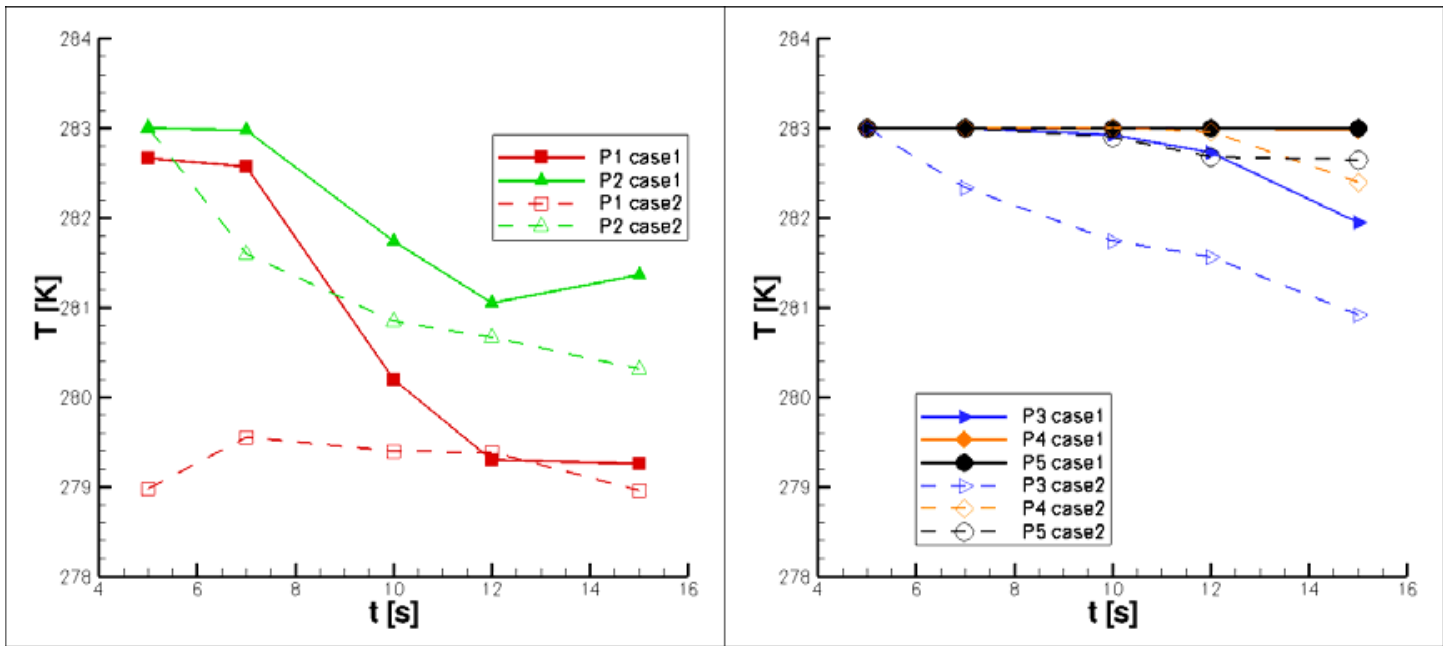

Figure 6. Temperature distribution along the central vertical axis (points P1 to P5 in Figure 1) 


\section{CONCLUSIONS}

The cases displayed are an example of the versatility and possibilities that numerical simulation provides for the study of the refrigerating cabinets of household frost-free refrigerators. Two different inlet opening distribution have been studied and their influence on the refrigerator performance evaluated.

Using the proposed inlet ports a more homogeneous temperature distribution in vertical direction is obtained. Furthermore, it is slightly more uniform in the horizontal direction. With case 2 configuration a more rapid cooling of larger regions within the refrigerating compartment is observed, i.e. after the same simulation time, the temperature has further decreased. However, it would be interesting to change the position of the two new inlet ports to the space between the third and the fourth shelves in order to reduce the temperature more rapidly in this region.

\section{ACKNOWLEDGEMENTS}

This research work has been financially funded in part by the Ministerio de Educación y Ciencia,Secretaría de Estado de Universidades e Investigación, Spain (ref. ENE2008-06667), and by a Generalitat de Catalunya Beatriu de Pinós postdoctoral fellowship (Ref. 2008 BP-A 00227).

\section{REFERENCES}

Borrell R., Lehmkuhl O., Soria M. and Oliva A., (2007), Schur complement methods for the solution of Poisson equation with unstructured meshes, Proceedings of the Parallel CFD 2007 Conference, 1-8.

Ding G.-L., Qiao H.-T., and Lu Z.-L., (2004), Ways to improve thermal uniformity inside a refrigerator, International Journal of Heat and Mass Transfer, 24, 1827-1840.

Fishpool G.M. and Leschziner M.A., (2009), Stability bounds for fractional-step schemes for the NavierStokes equations at high Reynolds number, Computers and Fluids, 38(6) 1289-1298.

Fukuyo K., Tanaami T. and Ashida H., (2003), Thermal uniformity and rapid cooling inside refrigerators, International Journal of Refrigeration, 26, 249-255.

Gupta J.K., Gopal M.R. and Chakraborty S., (2007), Modelling of a domestic frost-free refrigerator, International Journal of Refrigeration, 30, 311-322.

Jaramillo J., Rigola J., P'erez-Segarra C.D. and Oliet C., (2010), Numerical Study of Air Inside Refrigerating Compartment of Frost-free Domestic Refrigerators, Proceedings of the $13^{\text {th }}$ International Refrigeration and Air Conditioning Conference, 1-8.

Lehmkuhl O., Perez-Segarra C.D., Borrell R., Soria M. and Oliva A., (2007), TERMOFLUIDS: A new Parallel unstructured CFD code for the simulation of turbulent industrial problems on low cost PC Cluster, Proceedings of the Parallel CFD 2007 Conference, 1-8.

Meng X. and Yu B., (2009), Experimental research on air flow performance at supply-air openings in frost-free refrigerator by, Applied Thermal Engineering, 29, 3334-3339.

Nicoud F. and Ducros F., (1999), Subgrid-scale stress modeling based on the square of the velocity gradient tensor, Flow, Turbulence and Combustion, 62, 183-200.

Sagaut P., (2001), Large Eddy Simulation for Incompressible Flows, Springer-Verlag.

Smagorinsky J., (1963), General circulation experiments with the primitive equations, part. I: the basic experiment, Monthly Weather Rev., 91, 99-164.

Verstappen R.W.C.P. and Veldman A.E.P., (2003), Symmetry-preserving discretization of turbulent flow, Journal of Computational Physics, 187(1), 343-368. 